\title{
Frustration effects in rapidly rotating square and triangular optical lattices
}

\author{
T. P. Polak \\ Adam Mickiewicz University of Poznań, Faculty of Physics, Umultowska 85, 61-614 Poznań, Poland \\ T. K. Kopeć \\ Institute for Low Temperatures and Structure Research, \\ Polish Academy of Sciences, POB 1410, 50-950 Wroclaw 2, Poland
}

\begin{abstract}
We discuss the ground state of the two-dimensional Bose-Hubbard (BH) Hamiltonian, relevant for rotating gaseous Bose-Einstein condensates, by employing U (1) quantum rotor approach and the topologically constrained path integral that includes a summation over $\mathrm{U}(1)$ topological charge. We derive an effective quantum action for the $\mathrm{BH}$ model, which enables a non-perturbative treatment of the zero-temperature phase transition. We calculate the ground-state phase diagram, analytically deriving maximum repulsive energy for several rational values of the frustration rotation parameter $f=0,1 / 2,1 / 3,1 / 4$, and $1 / 6$ for the square and triangular lattice, which improves upon previous theoretical treatments. The ground state of the rotating Bose-Einstein condensates on a triangular lattice appears to be most stable against the effects of rotation. Performed calculations revealed strong dependence of the critical ratio of the kinetic energy to the repulsive on-site energy, that separates the global coherent from the insulating state, on topology of the lattice.

PACS numbers: 05.30.Jp, 03.75.Lm, 03.75.Nt
\end{abstract}

\section{INTRODUCTION}

The merging of atomic and condensed matter physics since the experimental realization of Bose-Einstein condensation $^{1}$ has opened exciting new perspectives for the creation of novel quantum states. Especially, systems of ultra-cold atoms confined in optical lattices 2 facilitate an experimental environment, where a rich variety of quantum many-body models can be implemented in a wide range of spatial dimensions, geometries, and particle interactions. Surprisingly, the quantum phase transitions in systems under uniform magnetic field can be also analyzed considering rotating Bose-Einstein condensates ${ }^{5}+7$ trapped in a two-dimensional $(2 D)$ lattice potential. In a frame of reference rotating about the $z$-axis with angular velocity $\Omega$ the kinetic term in Hamiltonian is equivalent to that of a particle of charge $q$ experiencing a magnetic field $B$ with $q B=2 m \Omega$, where $m$ is the mass of the particle ${ }^{89}$ This connection shows that the Coriolis force in the rotating frame plays the same role as the Lorentz force on a charged particle in an uniform magnetic field 1011 The presence of angular velocity induces vortices in the system described by the rotation frustration parameter $f\left(\equiv m a^{2} \Omega / \pi \hbar\right.$, with $a$ being the lattice spacing). The parameter $f$ can be also expressed in terms of the recoil energy $E_{R}$ as $f=\pi \hbar \Omega / 2 E_{R}$. Of special interest are cases when $f=p / q$, with $p$ and $q$ being the rational numbers. Frustration occurs in this system because two different area scales are in competition. One characteristic area is the unit cell $a$ of considered lattice. The other $\pi \hbar / m \Omega$ is associated with the rotation of the lattice. We can use the notion "magnetic field" and "rotation" interchangeably, assuming that a harmonic confinement potential is applied to cancel the centrifugal effects of rotation. Therefore, the nexus of condensed matter and optical physics is transparent (since the effects of magnetic field/rotation have the same mathematical structure) and different systems can mimic each other.

The progress in setups contrivance used for creation of a rotating optical lattice led to systems with different geometries like square $(\square)$ or triangular $(\triangle)$ that can be analyzed in the strongly interacting regime. ${ }^{6}$ Up to now two experimental strategies have been developed 12 The first one is based on direct imprinting a phase shift on the macroscopic wave function. 13 The second approach called "stirring" is an adaptation of the rotating bucket experiment to a gas of trapped bosons.14 16 The latter method fails when angular velocity is comparable to trapping frequency $\Omega \sim \omega_{\perp}$. However, several groups have found a way to circumvent the problem of the center of mass expulsion occurring at $\Omega \sim \omega_{\perp}$ and one can achieve $\Omega=1.05 \omega_{\perp} \cdot 17$ Mott-insulator (MI) - superfluid (SF) transition boundary obtained by using a Gutzwillertype variational wave function revealed the complexity of the dependence of phase boundary on the effective magnetic field/rotation, reflecting the self-similar properties of the single particle energy spectrum. 18 Mean field theory calculations determined that the linear eigenvalue equation characterizing the Mott lobe also characterizes the Hofstadter butterfly spectrum. From this authors determined an expression for the Mott-lobe boundary 19 Despite the several theoretical approaches to the problem of strongly interacting bosons in rotating lattices many questions still remain open and unsolved.

The aim of this work is to study the superfluid to Mottinsulator zero-temperature phase transition by means of the Bose-Hubbard (BH) model in two-dimensional rotating optical condensates with different geometries. We address the question of evolution of the ground state phase diagram for the system with various angular velocities for square and triangular lattices. The inherent difficulty of dealing with $\mathrm{BH}$ Hamiltonian appropriate for strongly 
correlated bosons originates from the non-perturbative nature of the model and the presence of rotation. To elucidate the quantum phase transition in optical lattices, where the kinetic energy scale is less than the dominating interaction energy and angular velocity is comparable to the recoil energy, we have adopted a theoretical approach for strongly interacting fermions 20 to the $\mathrm{BH}$ model in a way to include the effects of particle number fluctuations and make the qualitative phase diagrams more quantitative. ${ }^{21}$ To facilitate this task, we employ a functional integral formulation of the theory that enables to perform functional integration over fields defined on different topologically equivalent classes of the $\mathrm{U}(1)$ group, i.e., with different winding numbers. An inclusion of the winding numbers is unavoidable in order to obtain a proper phase diagram. The quantum rotor representation method we use is deeply rooted in the gauge symmetries of the model. We construct an invariant theory introducing an appropriate $\mathrm{U}(1)$ gauge transformation.

The outline of the paper is as follows In Sec. II we introduce the model Hamiltonian and the effects of rotation are discussed in Sec. III. Next, we derive an effective $U(1)$ action in the quantum rotor representation described in Sec. IV-VII. The aim of Sec. VIII is the presentation of the resulting phase diagrams for twodimensional square and triangular Bose-Hubbard systems in rotating frame and comparison of our results with several numerical and analytical calculations. Finally, Sec. IX summarizes our results. In the Appendix, we give an analytical derivation of density of states (DOS) in closed form for several rational values of $f=p / q$ in $\square$ and $\triangle$ lattice. Moreover, the connection between the DOS and Hofstadter butterfly is shown.

\section{MODEL}

In optical lattices the two main energy scales are set by the hopping amplitude proportional to $t$ (that sets the kinetic energy scale for bosons) due to the particles tunneling, and the on-site interaction $U>0$. For $t>U$ the phases of the superfluid order parameter on individual lattice sites are well defined. On the other hand, for sufficiently large repulsive energy $U$, the quantum phase fluctuations lead to complete suppression of the long-range phase coherence even at zero temperature. The competition between the kinetic energy, which is gained by delocalizing bosons over lattice sites and the repulsive interaction energy, which disfavors having more than one particle at any given site, can be modeled by the following quantum Bose-Hubbard Hamiltonian 22

$$
\mathcal{H}=\frac{U}{2} \sum_{\boldsymbol{r}} n_{\boldsymbol{r}}\left(n_{\boldsymbol{r}}-1\right)-\sum_{\left\langle\boldsymbol{r}, \boldsymbol{r}^{\prime}\right\rangle} t_{\boldsymbol{r} \boldsymbol{r}^{\prime}} a_{\boldsymbol{r}}^{\dagger} a_{\boldsymbol{r}^{\prime}}-\mu \sum_{\boldsymbol{r}} n_{\boldsymbol{r}}
$$

where $a_{\boldsymbol{r}}^{\dagger}$ and $a_{\boldsymbol{r}^{\prime}}$ stand for the bosonic creation and annihilation operators that obey the canonical commutation relations $\left[a_{\boldsymbol{r}}, a_{\boldsymbol{r}^{\prime}}^{\dagger}\right]=\delta_{\boldsymbol{r} \boldsymbol{r}^{\prime}}, n_{\boldsymbol{r}}=a_{\boldsymbol{r}}^{\dagger} a_{\boldsymbol{r}}$ is the boson number operator on the site $\boldsymbol{r}$, and the chemical potential $\mu$ controls the number of bosons. Here, $\left\langle\boldsymbol{r}, \boldsymbol{r}^{\prime}\right\rangle$ identifies summation over the nearest-neighbor sites. Furthermore, $t_{\boldsymbol{r} \boldsymbol{r}^{\prime}}$ is the hopping matrix element with dispersion $t_{\boldsymbol{k}}^{\square, \triangle}$.

\section{EFFECTS OF ROTATION}

In the fast rotation regime the physics of Bose-Einstein condensates is very reminiscent of that of charged particle in magnetic field. If the centrifugal term can be compensated by the trapping frequency in the plane perpendicular to the rotation axis $\left(\Omega=\omega_{\perp}\right)$, so only the Coriolis term is left, we have situation which formally is equivalent to the Lorentz force exerted by uniform magnetic field on charged particle. Experimentally a region of fast rotations up to $\Omega=1.05 \omega_{\perp} \frac{17}{17}$ that suits our theoretical predictions can be achieved.

An angular velocity enters the Hamiltonian Eq. (1) through the Peierls phase factor according to

$$
t_{\boldsymbol{r} \boldsymbol{r}^{\prime}} \rightarrow t_{\boldsymbol{r} \boldsymbol{r}^{\prime}} \exp \left(\frac{2 \pi i}{\kappa} \int_{\boldsymbol{r}}^{\boldsymbol{r}^{\prime}} \mathbf{A} \cdot d \boldsymbol{l}\right)
$$

where $\boldsymbol{A}(\boldsymbol{r})=\boldsymbol{\Omega} \times \boldsymbol{r}$ is the equivalent of a magnetic vector appears from the rotation and $\kappa=h / m$ is the quantum circulation unit. Thus, the phase shift on each site is determined by the vector potential $\mathbf{A}(\boldsymbol{r})$ and in typical experimental situations can be entirely ascribed to the external magnetic field/angular velocity. We assume throughout this paper that the model in Eq. (1) is defined on a lattice with lattice spacing $a=1$. From Eq. (2), it follows that the properties of the system will be periodic with a period corresponding to

$$
f=\pi \hbar \Omega / 2 E_{R}
$$

per plaquette. Of special interest are the values of the angular momentum which correspond to rational numbers of $f=1 / 2,1 / 3,1 / 4, \ldots$ Since all properties of the Hamiltonian Eq. (1) are invariant under $f \rightarrow-f$ and also under $f \rightarrow f+1$, it is sufficient to consider $f$ in the range $0<f<1 / 2$ that can be reached experimentally.

\section{DESCRIPTION OF THE METHOD}

We write the partition function of the system switching from the particle-number representation to the conjugate phase representation of the bosonic degrees of freedom using the bosonic path-integral over the complex fields $a_{\boldsymbol{r}}(\tau)$ depending on the "imaginary time" $0 \leq \tau \leq \beta \equiv$ $1 / k_{\mathrm{B}} T$ with $T$ being the temperature

$$
\begin{aligned}
\mathcal{Z}= & \int[\mathcal{D} \bar{a} \mathcal{D} a] \exp \left[-\int_{0}^{\beta} d \tau \mathcal{H}(\tau)\right. \\
& \left.-\sum_{\boldsymbol{r}} \int_{0}^{\beta} d \tau \bar{a}_{\boldsymbol{r}}(\tau) \frac{\partial}{\partial \tau} a_{\boldsymbol{r}}(\tau)\right] .
\end{aligned}
$$


We decouple the interaction term in Eq. (1) by a Gaussian integration over the auxiliary scalar potential fields

$$
V_{\boldsymbol{r}}(\tau)=V_{\boldsymbol{r} 0}+V_{\boldsymbol{r}}^{\prime}(\tau),
$$

with static

$$
V_{\boldsymbol{r} 0}=\beta^{-1} V_{\boldsymbol{r}}\left(\omega_{\nu}=0\right)
$$

and periodic part

$$
V_{\boldsymbol{r}}^{\prime}(\tau)=\beta^{-1} \sum_{\nu=1}^{+\infty} V_{\boldsymbol{r}}\left(\omega_{\nu}\right) \exp \left(i \omega_{\nu} \tau\right)+\text { c.c }
$$

where $\omega_{\nu}=2 \pi \nu / \beta(\nu=0, \pm 1, \pm 2, \ldots)$ is the BoseMatsubara frequency. We observe now that the $\mathrm{BH}$ Hamiltonian has a local U(1) gauge symmetry, when expressed in terms of the underlying boson variables. This points out a possibility of an emergent dynamical U(1) gauge field as a fluctuating complex field attached to bosonic variables, which is dynamically generated, by interacting bosons. Thus, the periodic part $V_{\boldsymbol{r}}^{\prime}(\tau) \equiv V_{\boldsymbol{r}}^{\prime}(\tau+\beta)$ couples to the local particle number through the Josephson-like relation $\dot{\phi}_{\boldsymbol{r}}(\tau)=V_{\boldsymbol{r}}^{\prime}(\tau)$, where

$$
\dot{\phi}_{\boldsymbol{r}}(\tau) \equiv \frac{\partial \phi_{\boldsymbol{r}}(\tau)}{\partial \tau}=e^{-\phi_{\boldsymbol{r}}(\tau)} \frac{1}{i} \frac{\partial}{\partial \tau} e^{\phi_{\boldsymbol{r}}(\tau)} .
$$

The quantity $\phi(\tau)$ is the $\mathrm{U}(1)$ phase field and satisfies the periodicity condition $\phi_{\boldsymbol{r}}(\beta)=\phi_{\boldsymbol{r}}(0)$ as a consequence of the periodic properties of the $V_{\boldsymbol{r}}^{\prime}(\tau)$ field in Eq. (7).

\section{PARAMETRIZATION OF THE BOSON FIELD AND THE ORDER PARAMETER}

We perform the local gauge transformation to the new bosonic variables

$$
\left[\begin{array}{l}
a_{\boldsymbol{r}}(\tau) \\
\bar{a}_{\boldsymbol{r}}(\tau)
\end{array}\right]=\left[\begin{array}{cc}
e^{i \phi_{\boldsymbol{r}}(\tau)} & 0 \\
0 & e^{-i \phi_{\boldsymbol{r}}(\tau)}
\end{array}\right]\left[\begin{array}{c}
b_{\boldsymbol{r}}(\tau) \\
\bar{b}_{\boldsymbol{r}}(\tau)
\end{array}\right]
$$

that removes the imaginary term $-i \int_{0}^{\beta} d \tau \dot{\phi}_{\boldsymbol{r}}(\tau) n_{\boldsymbol{r}}(\tau)$ from all the Fourier modes except at zero frequency. From the above we deduce bosons have a composite nature made of bosonic part $b_{\boldsymbol{r}}(\tau)$ and attached "flux" $\exp \left[i \phi_{\boldsymbol{r}}(\tau)\right]$. We parametrize the boson fields $b_{\boldsymbol{r}}(\tau)=$ $b_{0}+b_{\boldsymbol{r}}^{\prime}(\tau)$ and incorporate fully our calculations to the phase fluctuations governed by the gauge group $\mathrm{U}(1)$. Assuming nonfluctuating amplitude at low temperatures $b_{\boldsymbol{r}}(\tau)=b_{0}$, we drop the corrections, which was proved to be justified in the large $U / t$ limit we are interested in.2123 The calculation of $b_{0}$ is postponed to the next section.

It is very convenient to define the order parameter

$$
\Psi_{B} \equiv\left\langle a_{\boldsymbol{r}}(\tau)\right\rangle=\left\langle b_{\boldsymbol{r}}(\tau) \exp \left[i \phi_{\boldsymbol{r}}(\tau)\right]\right\rangle=b_{0} \psi_{B},
$$

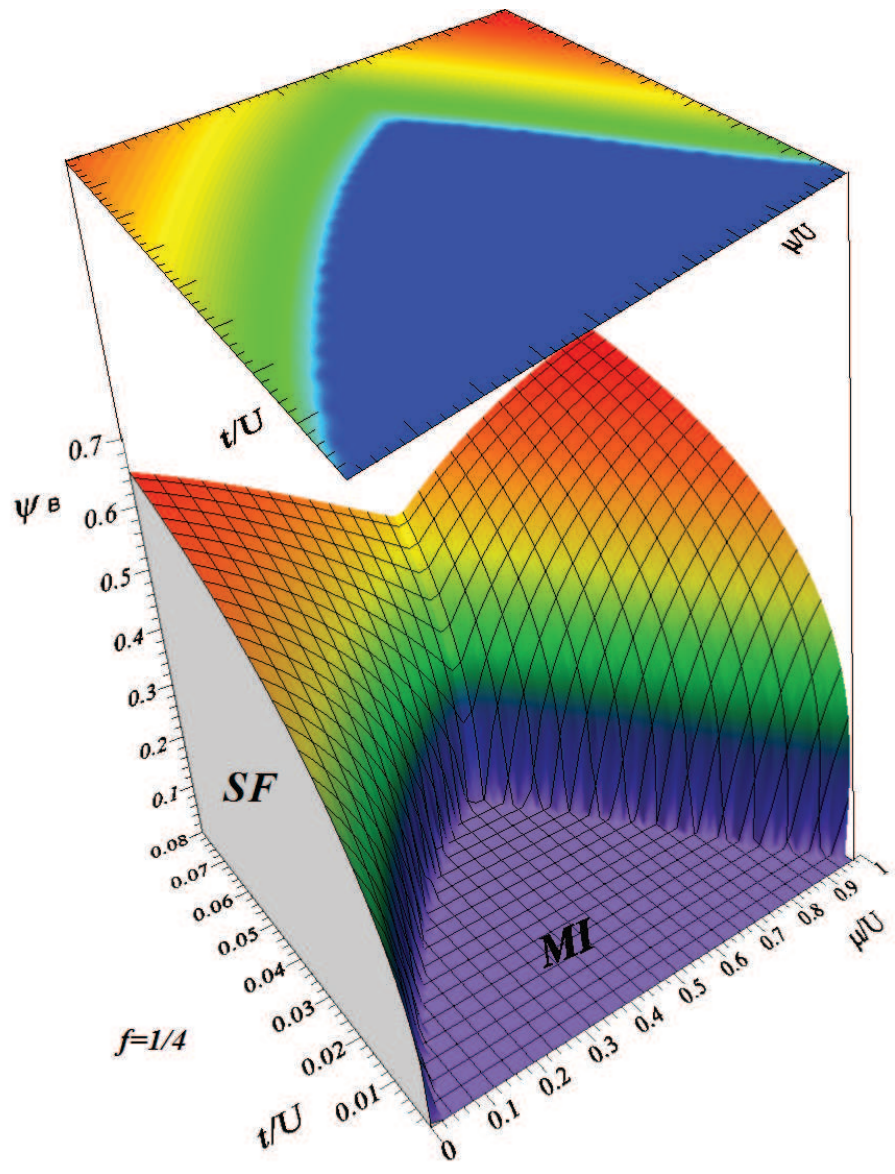

Figure 1: (Color online) The phase variable's part $\psi_{B}$ of the order parameter $\Psi_{B}$ Eq. 10 for rotating triangular lattice with the rotation frustration parameter $f=1 / 4$. Below the surface the phase coherent state (SF) takes place. Flat region means the incompressible Mott-insulator (MI) phase. The upper panel is the density plot of the surface in order to highlight the interaction $t / U$ - chemical potential $\mu / U$ dependence.

which signals the emergence of the superfluid phase and vanishes in the Mott-insulator state. The SF state is characterized by spontaneously breaking of the $\mathrm{U}(1)$ symmetry of the Bose-Hubbard Hamiltonian. Note, that a nonzero value of the amplitude $b_{0}$ in Eq. (10) is not sufficient for superfluidity. To achieve this, also the phase variables $\phi$ in Eq. 10 , must become stiff and coherent, which implies $\psi_{B} \neq 0$ (see Fig. 11 and Fig. 2). In the symmetry breaking state, with a finite expectation value of $a_{\boldsymbol{r}}(\tau)$, different phases $\phi_{1}, \phi_{2}, \ldots, \phi_{n}$ of the condensate lead to degenerate ground states (Fig. 2p. If we change the phase of the condensate in a large but finite region, then, locally, the system is still in one of the degenerate ground states. Slow changes of the phase result in the appearance of the low energy excitations that correspond to fluctuations among the degenerate states. Based on this picture we identify $b^{\prime}$ as high energy fast fluctuations (and drop them in calculations) contrary to the low 


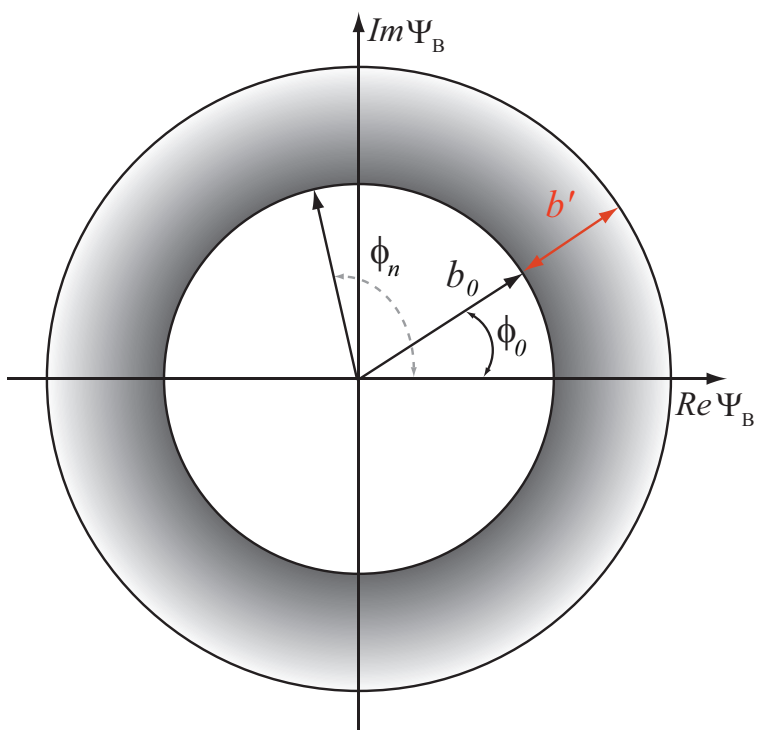

Figure 2: (Color online) The order parameter $\Psi_{B}$ can achieve nonzero value when both amplitude $b_{0}$ and phase $\phi$ are positive. The fluctuations of the amplitude $b^{\prime}$ in the Matsubara time in low-temperature limit $T \rightarrow 0$ are dropped in our approach.

energy fluctuations described by $\phi$.

\section{PHASE ONLY ACTION}

By integrating out the auxiliary static field $V_{\boldsymbol{r} 0}$ we calculate the partition function with an effective action expressed in the form of the propagator $\hat{G}$

$$
\mathcal{Z}=\int[\mathcal{D} \phi] e^{-\sum_{r} \int_{0}^{\beta} d \tau\left[\frac{1}{2 U} \dot{\phi}_{\boldsymbol{r}}^{2}(\tau)+\frac{1}{i} \frac{\bar{\mu}}{U} \dot{\phi}_{\boldsymbol{r}}(\tau)\right]+\operatorname{Tr} \ln \hat{G}^{-1}},
$$

where $\bar{\mu} / U=\mu / U+1 / 2$ is the shifted reduced chemical potential. In the above $\exp \left(-\operatorname{Tr} \ln \hat{G}^{-1}\right) \equiv \operatorname{det} \hat{G}$ and the determinant takes the form

$$
\begin{aligned}
\operatorname{det} \hat{G}= & \int[\mathcal{D} \bar{b} \mathcal{D} b] \exp \left\{-\sum_{\left\langle\boldsymbol{r}, \boldsymbol{r}^{\prime}\right\rangle} \int_{0}^{\beta} d \tau\right. \\
& \times \bar{b}_{\boldsymbol{r}}\left[\left(\frac{\partial}{\partial \tau}+\bar{\mu}\right) \delta_{\boldsymbol{r} \boldsymbol{r}^{\prime}}\right. \\
& \left.\left.-e^{i \phi_{\boldsymbol{r}}(\tau)} t_{\boldsymbol{r} \boldsymbol{r}^{\prime}} e^{-i \phi_{\boldsymbol{r}^{\prime}}(\tau)}\right] b_{\boldsymbol{r}}\right\} .
\end{aligned}
$$

The inverse of the propagator becomes

$$
\hat{G}^{-1}=\hat{G}_{0}^{-1}-K=\hat{G}_{0}^{-1}\left(1-K \hat{G}_{0}\right) .
$$

The explicit value of the amplitude $b_{0}$ in Eq. 10 can be obtained from minimization of the Hamiltonian $\partial \mathcal{H}\left(b_{0}\right) / \partial b_{0}=0$. Therefore, we write

$$
\hat{G}_{0}=b_{0}^{2} \equiv \frac{\sum_{\left\langle\boldsymbol{r}, \boldsymbol{r}^{\prime}\right\rangle} t_{\boldsymbol{r} \boldsymbol{r}^{\prime}}+\bar{\mu}}{U},
$$

$$
K=e^{i \phi_{\boldsymbol{r}}(\tau)} t_{\boldsymbol{r} \boldsymbol{r}^{\prime}} e^{-i \phi_{\boldsymbol{r}^{\prime}}(\tau)} .
$$

Expanding the trace of the logarithm we have

$$
\begin{aligned}
\operatorname{Tr} \ln \hat{G}^{-1}= & -\operatorname{Tr}\left(\ln \hat{G}_{0}\right)-\operatorname{Tr}\left(K \hat{G}_{0}\right) \\
& -\frac{1}{2} \operatorname{Tr}\left[(K \hat{G})^{2}\right]+\ldots
\end{aligned}
$$

with $\hat{G}_{0}$ and $K$ given by Eq. 14 and 15 . Finally the partition function Eq. (11) becomes

$$
\mathcal{Z}=\int[\mathcal{D} \phi] e^{-\mathcal{S}_{\text {phase }}[\phi]}
$$

with an effective action expressed only in the phase fields variable

$$
\begin{aligned}
\mathcal{S}_{\text {phase }}[\phi]= & \int_{0}^{\beta} d \tau\left\{\sum_{\boldsymbol{r}}\left[\frac{1}{2 U} \dot{\phi}_{\boldsymbol{r}}^{2}(\tau)+\frac{1}{i} \frac{\bar{\mu}}{U} \dot{\phi}_{\boldsymbol{r}}(\tau)\right]\right. \\
& \left.-\sum_{\left\langle\boldsymbol{r}, \boldsymbol{r}^{\prime}\right\rangle} e^{i \phi_{\boldsymbol{r}}(\tau)} J_{\boldsymbol{r} \boldsymbol{r}^{\prime}} e^{-i \phi_{\boldsymbol{r}^{\prime}}(\tau)}\right\}
\end{aligned}
$$

where the phase stiffness coefficient is given by $J_{\boldsymbol{r} \boldsymbol{r}^{\prime}}=$ $b_{0}^{2} t_{\boldsymbol{r} \boldsymbol{r}^{\prime}}$. The total time derivative Berry phase imaginary term in Eq. 18 is nonzero due to topological phase field configurations with $\phi_{\boldsymbol{r}}(\beta)-\phi_{\boldsymbol{r}}(0)=2 \pi m_{\boldsymbol{r}}\left(m_{\boldsymbol{r}}=\right.$ $0, \pm 1, \pm 2 \ldots)$ that result in topological ingredients to the correlator we will see below. Therefore, we concentrate on closed paths in the imaginary time $(0, \beta)$ labeled by the integer winding numbers $m_{\boldsymbol{r}}$. The path-integral

$$
\int[\mathcal{D} \phi] \ldots \equiv \sum_{\left\{m_{\boldsymbol{r}}\right\}} \int_{0}^{2 \pi}[\mathcal{D} \phi(0)] \int_{\phi_{\boldsymbol{r}}(0)}^{\phi_{\boldsymbol{r}}(\tau)+2 \pi m_{\boldsymbol{r}}}[\mathcal{D} \phi(\tau)] \ldots,
$$

includes a summation over $m_{\boldsymbol{r}}$ and in each topological sector the integration goes over the gauge potentials.

To proceed, we replace the phase degrees of freedom by the unimodular scalar complex field $\psi_{\boldsymbol{r}}$ which satisfies the quantum periodic boundary condition $\psi_{\boldsymbol{r}}(\beta)=\psi_{\boldsymbol{r}}(0)$. This can be conveniently done using the Fadeev-Popov method with Dirac delta functional resolution of unity 24 , where we take $\psi_{\boldsymbol{r}}$ as a continuous but constrained (on the average) variable to have the unimodular value

$$
\begin{aligned}
1 & =\int[\mathcal{D} \psi \mathcal{D} \bar{\psi}] \delta\left(\sum_{i}\left|\psi_{\boldsymbol{r}}(\tau)\right|^{2}-N\right) \\
& \times \prod_{\boldsymbol{r}} \delta\left(\psi_{\boldsymbol{r}}-e^{i \phi_{\boldsymbol{r}}(\tau)}\right) \delta\left(\bar{\psi}_{\boldsymbol{r}}-e^{-i \phi_{\boldsymbol{r}}(\tau)}\right),
\end{aligned}
$$

where $N$ is the number of lattice sites. Introducing the Lagrange multiplier $\lambda$, which adds the quadratic terms (in the $\psi_{\boldsymbol{r}}$ fields) to the action Eq. (18), we can solve for the constraint. The partition function can be rewritten to the form

$$
\mathcal{Z}=\int_{-i \infty}^{+i \infty}\left[\frac{\mathcal{D} \lambda}{2 \pi i}\right] e^{-N \beta \mathcal{F}(\lambda)}
$$


with the free energy density $\mathcal{F}=-\ln \mathcal{Z} / \beta N$ given by:

$$
\begin{aligned}
& \mathcal{F}=-\lambda-\frac{1}{N \beta} \ln \int[\mathcal{D} \psi \mathcal{D} \bar{\psi}] \exp \left\{\sum_{\boldsymbol{r} \boldsymbol{r}^{\prime}} \int_{0}^{\beta} d \tau d \tau^{\prime}\right. \\
& \times\left[\left(\mathcal{I}_{\boldsymbol{r}^{\prime}}+\lambda \delta_{\boldsymbol{r} \boldsymbol{r}^{\prime}}\right) \delta\left(\tau-\tau^{\prime}\right)\right. \\
& \left.\left.-\gamma_{\boldsymbol{r} \boldsymbol{r}^{\prime}}\left(\tau, \tau^{\prime}\right)\right] \psi_{\boldsymbol{r}} \bar{\psi}_{\boldsymbol{r}^{\prime}}\right\} \text {, }
\end{aligned}
$$

where $\mathcal{I}_{\boldsymbol{r} \boldsymbol{r}^{\prime}}=1$ if $\boldsymbol{r}, \boldsymbol{r}^{\prime}$ are the nearest neighbors and equals zero otherwise, and

$$
\gamma_{\boldsymbol{r} \boldsymbol{r}^{\prime}}\left(\tau, \tau^{\prime}\right)=\left\langle\exp \left\{-i\left[\phi_{\boldsymbol{r}}(\tau)-\phi_{\boldsymbol{r}^{\prime}}\left(\tau^{\prime}\right)\right]\right\}\right\rangle
$$

is the two-point phase correlator associated with the order parameter field, where $\langle\ldots\rangle$ denotes averaging with respect to the action in Eq. (18). The final form of the correlator with topological contribution (summation over integer winding numbers)

$$
\begin{aligned}
\gamma_{\boldsymbol{r} \boldsymbol{r}^{\prime}}\left(\tau, \tau^{\prime}\right) & =\frac{\delta_{\boldsymbol{r} \boldsymbol{r}^{\prime}} \exp \left(\frac{U}{2}\left|\tau-\tau^{\prime}\right|\right)}{\sum_{\left\{m_{\boldsymbol{r}}\right\}} \exp \left[-\frac{U \beta}{2}\left(m_{\boldsymbol{r}}+\frac{\bar{\mu}}{U}\right)^{2}\right]} \\
& \times \sum_{\left\{m_{\boldsymbol{r}}\right\}}\left\{\exp \left[-\frac{U \beta}{2}\left(m_{\boldsymbol{r}}+\frac{\bar{\mu}}{U}\right)^{2}\right]\right. \\
& \left.\times \exp \left[-U\left(m_{\boldsymbol{r}}+\frac{\bar{\mu}}{U}\right)\left(\tau-\tau^{\prime}\right)\right]\right\}
\end{aligned}
$$

after Fourier transform, can be written as

$$
\gamma\left(\omega_{\nu}\right)=\frac{1}{\mathcal{Z}_{0}} \frac{4}{U} \sum_{m=-\infty}^{+\infty} \frac{\exp \left[-\frac{U \beta}{2}\left(m+\frac{\bar{\mu}}{U}\right)^{2}\right]}{1-4\left(m+\frac{\bar{\mu}}{U}-i \frac{\omega_{\nu}}{U}\right)^{2}}
$$

where

$$
\mathcal{Z}_{0}=\sum_{m=-\infty}^{+\infty} \exp \left[-U \beta(m+\bar{\mu} / U)^{2} / 2\right]
$$

is the partition function for the set of quantum rotors. The form of Eq. 25 assures the periodicity in the imaginary time with respect to $\bar{\mu} / U=\mu / U+1 / 2$ which emphasizes the special role of its integer values. The action Eq. 18, with the topological contribution Eq. 24, after Fourier transform, is written as

$$
\mathcal{S}_{\mathrm{eff}}[\psi, \bar{\psi}]=\frac{1}{N \beta} \sum_{\mathbf{k} \nu} \bar{\psi}_{\mathbf{k} \nu} \Gamma_{\mathbf{k}}^{-1}\left(\omega_{\nu}\right) \psi_{\mathbf{k} \nu}
$$

where $\Gamma_{\mathbf{k}}^{-1}\left(\omega_{\nu}\right)=\lambda-J_{\mathbf{k}}+\gamma^{-1}\left(\omega_{\nu}\right)$ is the inverse of the propagator.

\section{CRITICAL LINES}

Within the phase coherent state the order parameter $\psi_{B}$ is evaluated in the thermodynamic limit $N \rightarrow \infty$ by the saddle point method $\delta \mathcal{F} / \delta \lambda=0$ and the unimodular condition of the $\mathrm{U}(1)$ phase variables translates into the equation

$$
1-\psi_{B}^{2}=\frac{1}{N \beta} \sum_{\mathbf{k}, \nu} \frac{1}{\lambda-J_{\mathbf{k}}+\gamma^{-1}\left(\omega_{\nu}\right)} .
$$

The phase boundary is determined by the divergence of the order parameter susceptibility $\Gamma_{\mathbf{k}=0}\left(\omega_{\nu=0}\right)=0$

$$
\lambda_{0}-J_{\mathrm{p} / \mathrm{q}}^{\max }+\gamma^{-1}\left(\omega_{\nu=0}\right)=0
$$

which determines the critical value of the Lagrange parameter $\lambda=\lambda_{0}$, that stays constant in the whole global coherent phase. To proceed, it is desirable to introduce the density of states for a $2 D$ lattice in the rotating frame in the form

$$
\rho_{p / q}^{\square, \Delta}(\xi)=\frac{1}{N} \sum_{\boldsymbol{k}} \delta\left(\xi-\frac{t_{\boldsymbol{k}}^{\square, \triangle}}{t}\right)
$$

with $t_{\boldsymbol{k}}^{\square, \triangle}$ being the Fourier transform of the hopping matrix elements. In this context the quantity $J_{\mathrm{p} / \mathrm{q}}^{\max }$ in Eq. (29) represents the maximum of the spectrum described by the DOS Eq. 30. The problem of computing of $\rho_{p / q}^{\square, \Delta}(\xi)$ reduces effectively to the solution of the Harper equation relevant, e.g., to tight binding electrons on a two-dimensional lattice with an uniform magnetic flux per unit plaquette. In the Appendix, we give an analytical derivation of $\rho_{p / q}^{\square, \Delta}(\xi)$ in closed form for several rational values of $p / q$. With the help of the above and after summation over Bose-Matsubara frequency $\omega_{\nu}$, the superfluid state order parameter becomes

$$
1-\psi_{B}^{2}=\frac{1}{2} \int_{-\infty}^{+\infty} \frac{\rho_{p / q}^{\square, \Delta}(\xi) d \xi}{\sqrt{2 \bar{\xi}\left(2 z \frac{t}{U}+\frac{\mu}{U}+\frac{1}{2}\right) \frac{t}{U}+v^{2}\left(\frac{\mu}{U}\right)}}
$$

In Eq. (31) $v(\mu / U)=\operatorname{frac}(\mu / U)-1 / 2$, where frac $(x)=$ $x-[x]$ is the fractional part of the number and $[x]$ is the floor function which gives the greatest integer less than or equal to $x ; \bar{\xi}=J_{\mathrm{p} / \mathrm{q}}^{\max }-\xi$ with $J_{\mathrm{p} / \mathrm{q}}^{\max }$ stands for the maximum value of the dispersion spectrum $t_{\boldsymbol{k}}^{\square, \triangle}$ and $z$ is the lattice coordination number.

\section{PHASE DIAGRAMS}

The zero-temperature phase diagram of the homogeneous Bose-Hubbard model Eq. (1) can be calculated from Eq. (31) and is shown schematically in Fig. 3 as a function of $t / U$, with the density controlled by a chemical potential $\mu / U$. At $U / t \rightarrow 0$, the kinetic energy dominates and the ground state is a delocalized superfluid, described by nonzero value of the superfluid order parameter $\Psi_{B} \neq 0$. At small values of $t / U$, interactions dominate and one obtains a series of MI lobes with fixed integer filling $n_{B}=1,2, \ldots{ }^{21 \mid 22}$ The transition be- 


\begin{tabular}{c|c|c|c|c|c}
\hline \hline & QMC & DPT & MFT & PA & QRA \\
\hline$x_{0}^{\square}$ & $0.05974(3)$ & 0.05909 & 0.043 & 0.059 & 0.06719 \\
\hline \hline
\end{tabular}

Table II: Comparison of the maximum of the critical value for $t / U$ (as a function of the normalized chemical potential $\mu / U$ ) at the tip of the first $\left(n_{B}=1\right)$ MI lobe for the square lattice with several numerical (QMC - quantum Monte-Carlo ${ }^{25]}$ DPT - diagrammatic perturbation theory ${ }^{26}$ ) and analytical works (MFT - mean-field theory, ${ }^{[27} \mathrm{PA}$ - Padé analysis, ${ }^{[2]} \mathrm{QRA}$ - our calculations using quantum rotor approach).

tween the SF and MI phases is associated with the loss of long-range order. Let us introduce the notation for the maximum of the critical value for parameter $t / U$ (as a function of the normalized chemical potential $\mu / U$ ) at the tip of the first $\left(n_{B}=1\right)$ MI lobe for different lattices and frustration parameters $f$ as follows

$$
x_{f}^{\square, \Delta} \equiv \max \left\{\left(\frac{t}{U}\right)_{\text {crit }}\right\}_{f}^{\square, \Delta} .
$$

In Table II we compare values of the $x_{0}^{\square}$ resulting from several numerica $\sqrt{25 \mid 26}$ and analytical studies. $27 \sqrt{28}$ We found them in good agreement however, mean-field theory calculations of the $\mathrm{BH}$ model underestimate $x_{0}^{\square}$ and in the quantum rotor approach there is a slight upward trend of the boundary towards higher critical values of parameter $x_{0}^{\square}$ than obtained from numerical calculations. The ground state of the rotating Bose-Einstein condensates on a triangular lattice appears to be most stable against the effect of rotation (see Fig. 3). The stability comes from the higher values of the repulsive energy for the triangular lattice. However, if the rotation frustration parameter is equal $f=1 / 3$ and $1 / 2$ the ratio $x_{f}^{\triangle} / x_{f}^{\square}$ of the energy needed to cause loss of the global coherent state changes character and is higher for triangular lattice unlike the cases with $f=0,1 / 6$ and $1 / 4$ (see Fig. 4 and Fig. 57. In the above we choose $\mu / U=1 / 2$ because the transition at integer density belongs to the universality class of the $2+1$ dimensional $X Y$ model by contrast to transition if one cross SF-MI phase boundary by variations in the chemical potential. Behavior of the maximum repulsive energy $x_{f}^{\square, \triangle}$ in the rotating system with $f \neq 0$ taken for special value of the $\mu / U=1 / 2$ is non-monotonical in both square and triangular lattice (Fig. 6 and Fig. 7). While critical values for $x_{f}^{\square, \triangle}$ are different for various topologies of the system, the transition seen in the time-of-flight images occurs rather rapidly with increasing lattice depth. ${ }^{3}$ Because the experimental parameter $V_{0} / E_{R}\left(V_{0}\right.$ is the maximum value of the lattice depth), depends logarithmically on $U / t$, the small changes of the dimensionless depth of the optical lattice can cover a wide range of the phase diagram. In order to verify the calculated phase boundaries experimentally one shall be able to obtain the higher resolution than required to distinguish the $n_{B}=1$ from $n_{B}=2$ tran-

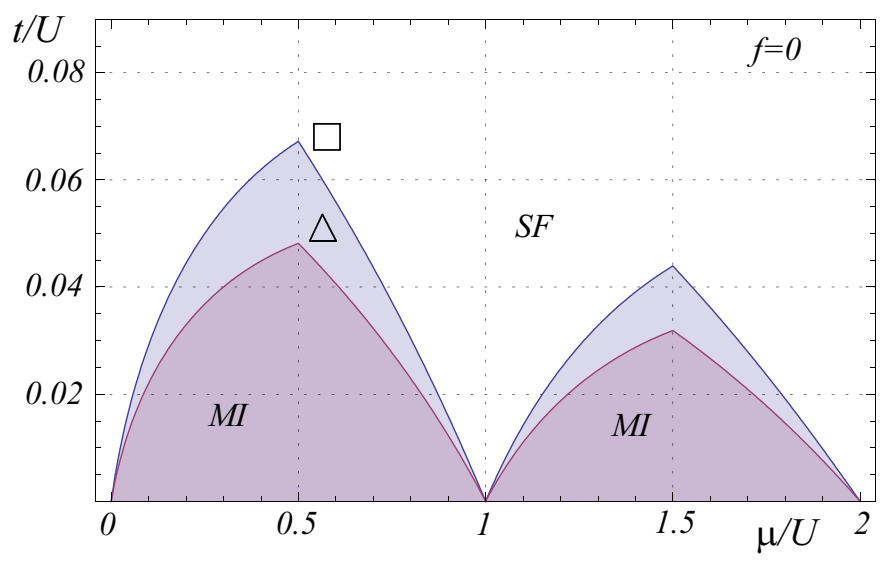

Figure 3: (Color online) Phase diagram for square $\square$ and triangular $\triangle$ lattice (number of particles per lattice site is $n_{B}=1$ inside the first and $n_{B}=2$ inside the second lobe respectively) with no rotation $f=0$. Within the lobes the MI phase takes place with $\Psi_{B}=0$ (see also Fig. 1).

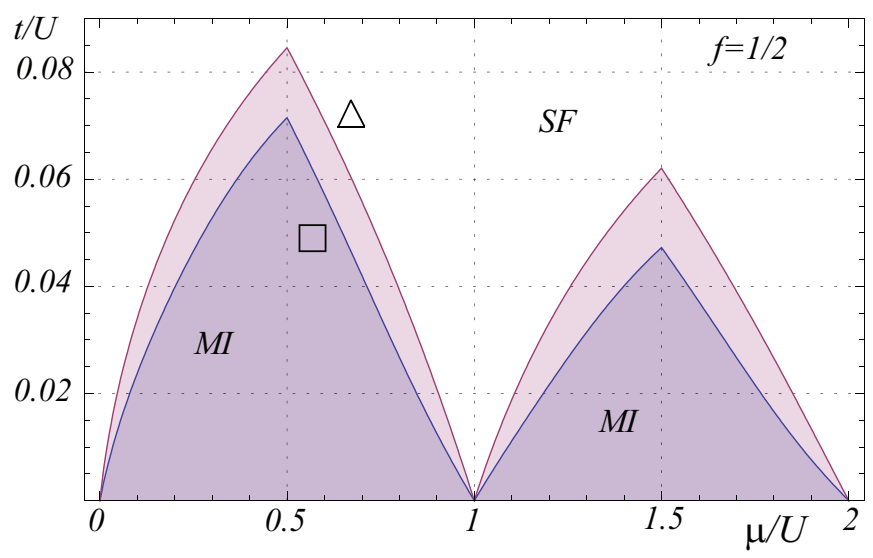

Figure 4: (Color online) Phase diagram for square $\square$ and triangular $\triangle$ lattice (number of particles per lattice site is $n_{B}=1$ inside the first and $n_{B}=2$ inside the second lobe respectively) with rotation frustration parameter $f=1 / 2$. Within the lobes the MI phase takes place with $\Psi_{B}=0$ (see also Fig. 11.

sition with $x_{0}^{\square, \triangle}\left(n_{B}=2\right) / x_{0}^{\square, \triangle}\left(n_{B}=1\right) \approx 0.65$. With increasing number of particles per lattice site $\left(n_{B} \rightarrow \infty\right)$, the system possesses an exact particle-hole symmetry thus, there is no difference between left $\bar{\mu} / U=n-\epsilon$ and right $\bar{\mu} / U=n+\epsilon$ branch of the $n$-th lobe, where $\epsilon \leq 0.5$.

\section{CONCLUSIONS}

The physics of strongly correlated bosonic systems is the competition between two tendencies of the bosons to spread out as a wave and to localize as a particle combined with a frustration caused by rotation. We presented a field-theoretic study of the ground-phase dia- 


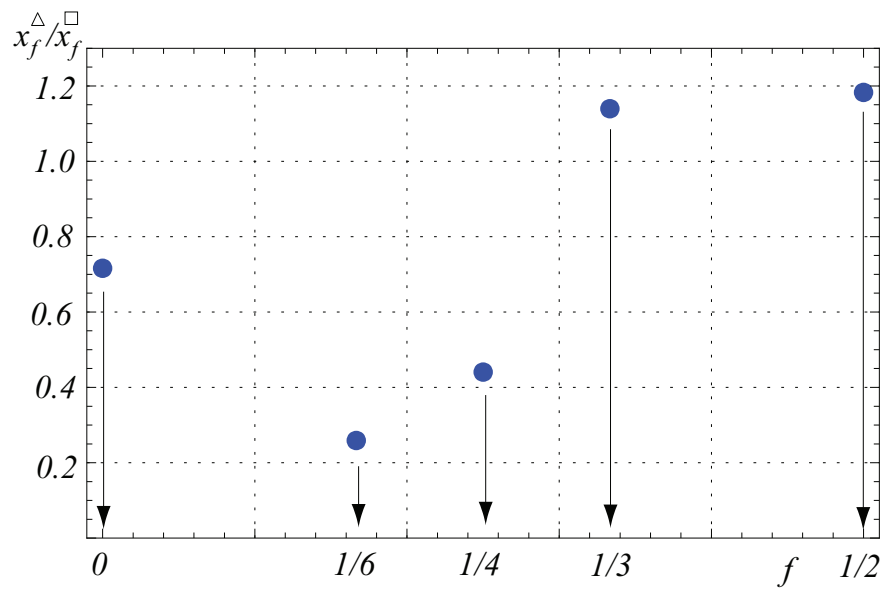

Figure 5: (Color online) The maximum of the critical value for $t / U$ parameter (as a function of the normalized chemical potential $\mu / U)$ at the tip of the first $\left(n_{B}=1\right)$ MI lobe $x_{f}^{\triangle} / x_{f}^{\square}$ for rotating triangular to square lattice.

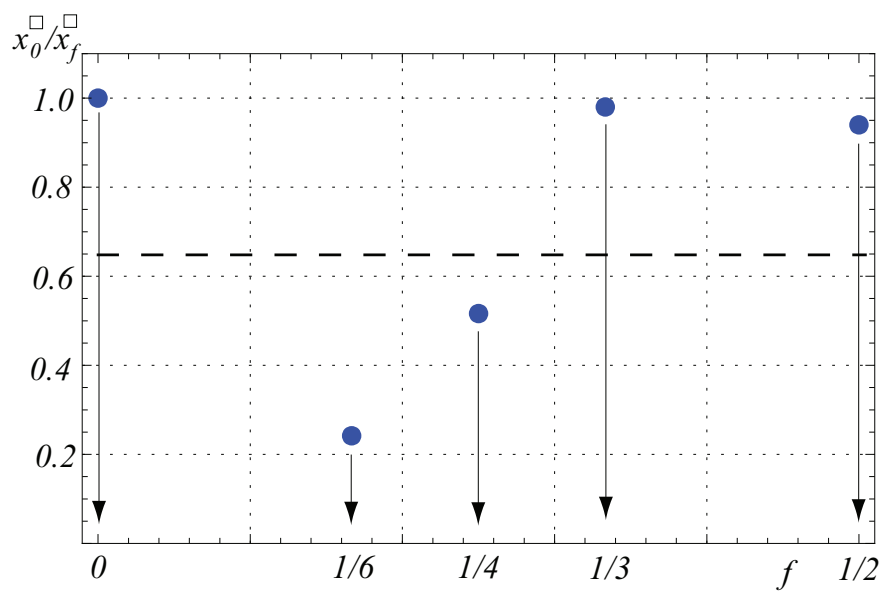

Figure 6: (Color online) The maximum of the critical value for $t / U$ parameter (as a function of the normalized chemical potential $\mu / U)$ at the tip of the first $\left(n_{B}=1\right)$ MI lobe $x_{0}^{\square} / x_{f}^{\square}$ for rotating square lattice. The vertical dashed line marks the ratio of the maximum of the critical value for $t / U$ parameter for the second to first lobe $x_{0}^{\square}\left(n_{B}=2\right) / x_{0}^{\square}\left(n_{B}=1\right)$.

gram in quantum two-dimensional gaseous Bose-Einstein condensates where mentioned emulation takes place. We calculated the phase diagram using the quantum rotor approach with exactly evaluated density of states for twodimensional lattices with rational magnetic flux/rotation frustration parameter $f=p / q$ for a number of values $f=1 / q$. In systems that are in the global coherent state at $f=0$, but with the ratio $t / U$ close to the critical value $(t / U)_{\text {crit }}$, a rotation can be used to drive the condensates into the MI state (Fig. 3). We compare the maximum of the critical value for $t / U$ parameter (as a function of the normalized chemical potential $\mu / U$ ) at the tip of the first $\left(n_{B}=1\right)$ MI lobe for square lattice with several numerical and analytical works and found them in a good

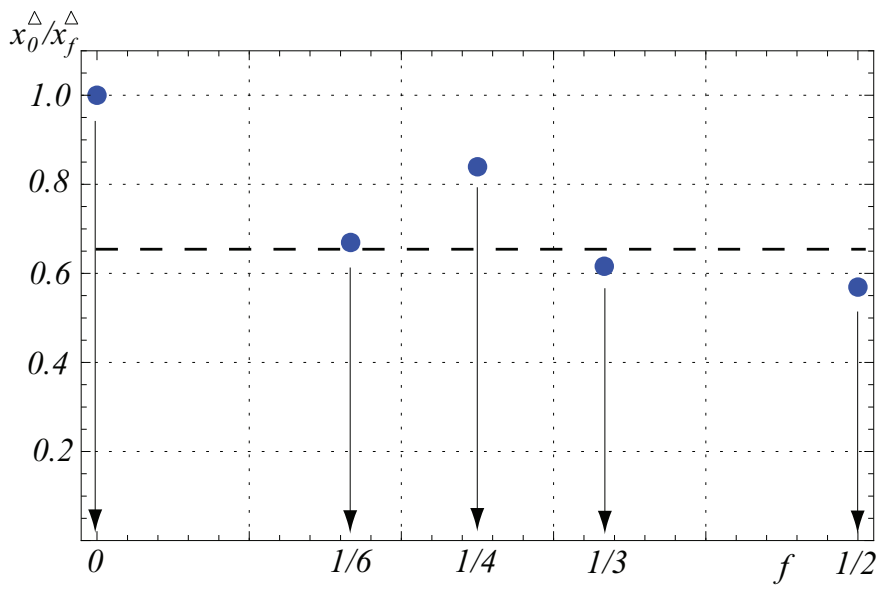

Figure 7: (Color online) The maximum of the critical value for $t / U$ parameter (as a function of the normalized chemical potential $\mu / U)$ at the tip of the first $\left(n_{B}=1\right)$ MI lobe $x_{0}^{\triangle} / x_{f}^{\triangle}$ for rotating triangular lattice. The vertical dashed line marks the ratio of the maximum of the critical value for $t / U$ parameter for the second to first lobe $x_{0}^{\triangle}\left(n_{B}=2\right) / x_{0}^{\triangle}\left(n_{B}=1\right)$.

agreement. Note that the dependence of the $x_{f}^{\square, \triangle} / x_{0}^{\square, \triangle}$ from frustration parameter $f$ is non-monotonical (Fig. 6 and Fig. 7p. The critical values of the energy needed to drive a rotating condensate out of a global coherent state change by varying the frustration parameter and strongly depends on topology of the lattice.

The nice feature of presented approach, described in details above, is that all the expressions and handling are analytic. It is also worth to notice that we provide an exact formulas for density of states that can be very useful in various situations whenever the magnetic field/rotation is applied to the physical system. To our knowledge the analytical expressions for DOS for triangular lattice were not known in the literature. Notice, we consider only the limit $T \rightarrow 0$ since in twodimensional systems with a continuous symmetry the long-range order is destroyed by the quantum fluctuations at finite temperature 29 Moreover, we want to emphasize that our approach cannot be used for analysis of the Berezinski-Kosterlitz-Thouless transitions since it is appropriate only for physical systems where long-range order appears.

\section{Acknowledgments}

We thank R. Micnas and T. A. Zaleski for fruitful and stimulating discussions and R. W. Chhajlany for careful reading of the manuscript.

\section{Appendix A: Density of States}

In this appendix we give the explicit formulas for the density of states Eq. 30 for square and triangular lat- 


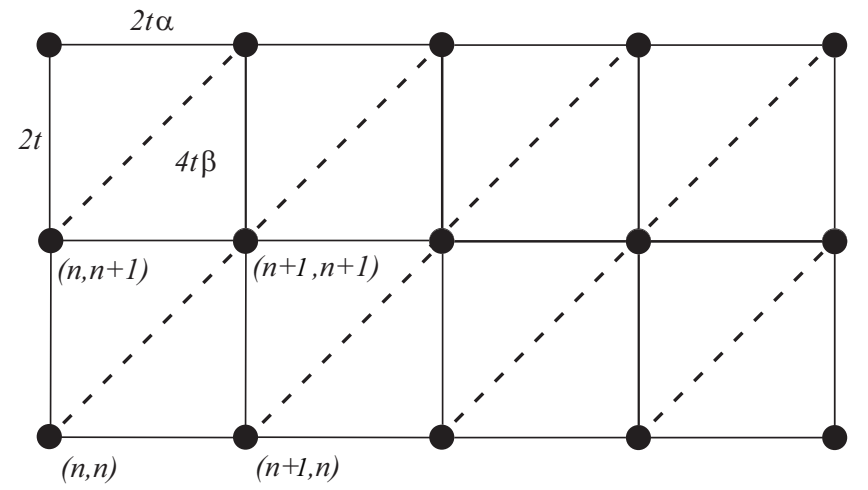

Figure 8: The lattice we used in our calculations is topologically equivalent to triangular structure. It appears from square lattice when we add bonds between next-nearest neighbors $(n, n)$ and $(n+1, n+1)$, thus if $\phi$ is the flux per plaquette then for triangular lattice we have $\phi / 2$.

tice structures with uniform magnetic field/rotation. The provided analytical expression can be advantageous in evaluating sums over momenta in Eq. 28. Moreover, the connection between the DOS and Hofstadter butterfly will be shown.

We start from the dispersion relevant for a lattice that can be viewed as more general than square and triangular since it includes both cases (Fig. 8)

$$
t_{\boldsymbol{k}}=-2 t\left[\cos k_{1}+\alpha \cos k_{2}+2 \beta \cos \left(k_{1}+k_{2}\right)\right] .
$$

Regarding the above parameters $\alpha=0$ and $\beta=0$ lead to $1 D$ chain; $\alpha=1$ and $\beta=0$ to the square lattice

$$
t_{\boldsymbol{k}}^{\square}=-2 t\left(\cos k_{1}+\cos k_{2}\right)
$$

and finally $\alpha=1$ and $\beta=0.5$ to the triangular structure

$$
t_{\boldsymbol{k}}^{\triangle}=-2 t\left[\cos k_{1}+\cos k_{2}+\cos \left(k_{1}+k_{2}\right)\right] .
$$

The different sign of the $\beta$ parameter changes the parity of the DOS that will be very useful in some cases of triangular lattice in rotating frame. Performing integration over momenta

$$
\rho(\alpha, \beta, \xi)=\int_{-\pi}^{+\pi} \frac{d^{2} \mathbf{k}}{(2 \pi)^{2}} \delta\left[\xi-\cos k_{1}-\alpha \cos k_{2}-2 \beta \cos \left(k_{1}+k_{2}\right)\right]
$$

we get:

$$
\rho(\alpha, \beta, \xi)=\left\{\begin{array}{ccc}
\frac{1}{\pi^{2} \sqrt{\alpha+\beta \xi}} \mathbf{K}\left(\sqrt{\frac{(1+\alpha)^{2}-(\xi-\beta)^{2}}{4(\alpha+\beta \xi)}}\right) & \text { for } \begin{array}{c}
1+\alpha+\beta \geq \xi \geq 1-\alpha-\beta \\
-1+\alpha-\beta \geq \xi>-1-\alpha+\beta
\end{array} \\
\frac{2}{\pi^{2} \sqrt{(1+\alpha)^{2}-(\xi-\beta)^{2}}} \mathbf{K}\left(\sqrt{\frac{4(\alpha+\beta \xi)}{(1+\alpha)^{2}-(\xi-\beta)^{2}}}\right) & \text { for } \quad 1-\alpha-\beta \geq \xi \geq-1+\alpha-\beta
\end{array}\right.
$$

where $\mathbf{K}(k)=\int_{0}^{\pi} d x\left(1-k^{2} \sin ^{2} x\right)^{-1 / 2}$ is the complete elliptic function of the first kind $\underline{32}$

\section{Appendix B: Square lattice in rotating frame}

The effects of homogeneous magnetic field/rotation on particles have many interesting features. While energy levels are quantized into Landau levels in $2 D$ uniform space, a very rich structure appears e.g. Hofstadter butterfly $\sqrt{30}$, when the lattice geometry is taken into account 31 If one uses the Landau gauge $A(0, x, 0)$ then the dispersion $t_{\boldsymbol{k}}^{\square}$ for a square lattice of spacing $a=1$ with rotation frustration parameter $f=p / q$ is given by

$$
\operatorname{det}\left(\begin{array}{ccccc}
M_{1} & -e^{-i k_{1}} & \cdots & 0 & -e^{-i k_{1}} \\
-e^{i k_{1}} & M_{2} & -e^{-i k_{1}} & 0 & 0 \\
\vdots & -e^{i k_{1}} & M_{3} & \ddots & 0 \\
0 & 0 & \ddots & \ddots & -e^{-i k_{1}} \\
-e^{-i k_{1}} & 0 & 0 & -e^{i k_{1}} & M_{n}
\end{array}\right)=0
$$

where

$$
M_{n}=-t_{\boldsymbol{k}}^{\square}-2 \cos \left(k_{2}+2 \pi f n\right) .
$$

Equation (B1) is known as Harper's equation and has been studied extensively. If integers $p$ and $q$ are chosen to represent the angular velocity (with no common factor in $p$ and $\mathrm{q}$ ), then the dependence on the wave vector always appears through the generalized structure factor $\gamma_{n}^{\square}=\cos n k_{1}+\cos n k_{2}$. The density of states given by Eq. 30 can be obtained by computing energy bands $t_{\boldsymbol{k}}^{\square}$ from the eigenvalue equation (B1) see Table IV 


\begin{tabular}{c|c|c}
\hline \hline$p$ & $q$ & $t_{k} \equiv t_{k}^{\square}$ \\
\hline 1 & 2 & $t_{k}^{2}-4-2 \gamma_{2}^{\square}=0$ \\
\hline 1 & 3 & $t_{k}^{3}-6 t_{k}+2 \gamma_{3}^{\square}=0$ \\
\hline 1 & 4 & $t_{k}^{4}-8 t_{k}^{2}+4-2 \gamma_{4}^{\square}=0$ \\
\hline 1 & 6 & $t_{k}^{6}-12 t_{k}^{4}+24 t_{k}^{2}-4-2 \gamma_{6}^{\square}=0$ \\
\hline \hline
\end{tabular}

Table IV: Energy dispersion for rotating square lattice for values $f=p / q$ used in calculations.

The calculation of the exact formulae for DOS is straightforward, although for large values of $q$ may only be done numerically. However, for a number of $q$ values of interest it can be calculated analytically with a closedform expression for $\rho_{p / q}^{\square}(\xi)$ as the end result. Below we list these cases.

\section{Square lattice without rotation - $f=0$}

In the case of zero rotation the density of states for the square $2 D$ lattice reads simply

$$
\rho_{0}^{\square}(\xi)=\rho(1,0, \xi) .
$$

2. Rotating square lattice with $f=1 / 2$

$$
\rho_{1 / 2}^{\square}(\xi)=\frac{|\xi|}{2} \rho_{0}^{\square}\left[\frac{1}{2}\left(\xi^{2}-4\right)\right] \Theta\left(8-\xi^{2}\right) .
$$

In the above $\Theta(x)$ is the unit step function.

3. Rotating square lattice with $f=1 / 3$

$$
\begin{aligned}
\rho_{1 / 3}^{\square}(\xi) & =\frac{1}{4 \sqrt{2}}\left|\left(\xi^{2}-2\right) \sqrt{\xi^{2}-8}\right| \rho_{0}^{\square}\left[\frac{1}{2} \xi\left(\xi^{2}-6\right)\right] \\
& \times\left\{\left|\sec \left(\varphi+\frac{\pi}{2}\right)\right|\left[\Theta(\xi+1+\sqrt{3})-\Theta\left(6-\xi^{2}\right)-\Theta(\xi-1-\sqrt{3})\right]\right. \\
+ & \sec \left(\varphi+\frac{\pi}{6}\right)[\Theta(\xi+\sqrt{6})-\Theta(\xi+2)+\Theta(\xi)-\Theta(\xi+1-\sqrt{3})] \\
+ & \left.\sec \left(\varphi-\frac{\pi}{6}\right)[\Theta(\xi-1+\sqrt{3})-\Theta(\xi)+\Theta(\xi-2)-\Theta(\xi-\sqrt{6})]\right\}, \\
\varphi & =\frac{1}{3} \arctan \left(\frac{\sqrt{32-\xi^{2}\left(\xi^{2}-6\right)^{2}}}{\xi\left(\xi^{2}-6\right)}\right) .
\end{aligned}
$$

4. Rotating square lattice with $f=1 / 4$

$$
\begin{aligned}
\rho_{1 / 4}^{\square}(\xi) & =\frac{1}{2}\left|\xi^{2}-4\right| \rho_{0}^{\square}\left[\frac{1}{2}\left(\xi^{4}-8 \xi^{2}+4\right)\right]\left\{\sqrt{4+|\xi-4|}\left[\Theta\left(8-\xi^{2}\right)-\Theta\left(4+2 \sqrt{2}-\xi^{2}\right)\right]\right. \\
& \left.+\sqrt{4-\left|\xi^{2}-4\right|} \Theta\left(4-2 \sqrt{2}-\xi^{2}\right)\right\} .
\end{aligned}
$$

\section{Rotating square lattice with $f=1 / 6$}

$$
\rho_{1 / 6}^{\square}(\xi)=\frac{1}{4 \sqrt[4]{2}}\left|\left(\xi^{4}-8 \xi^{2}+8\right) \sqrt{16+8 \xi^{2}-\xi^{4}}\right| \rho_{0}^{\square}\left[\frac{1}{2}\left(\xi^{6}-12 \xi^{4}+24 \xi^{2}-4\right)\right]
$$



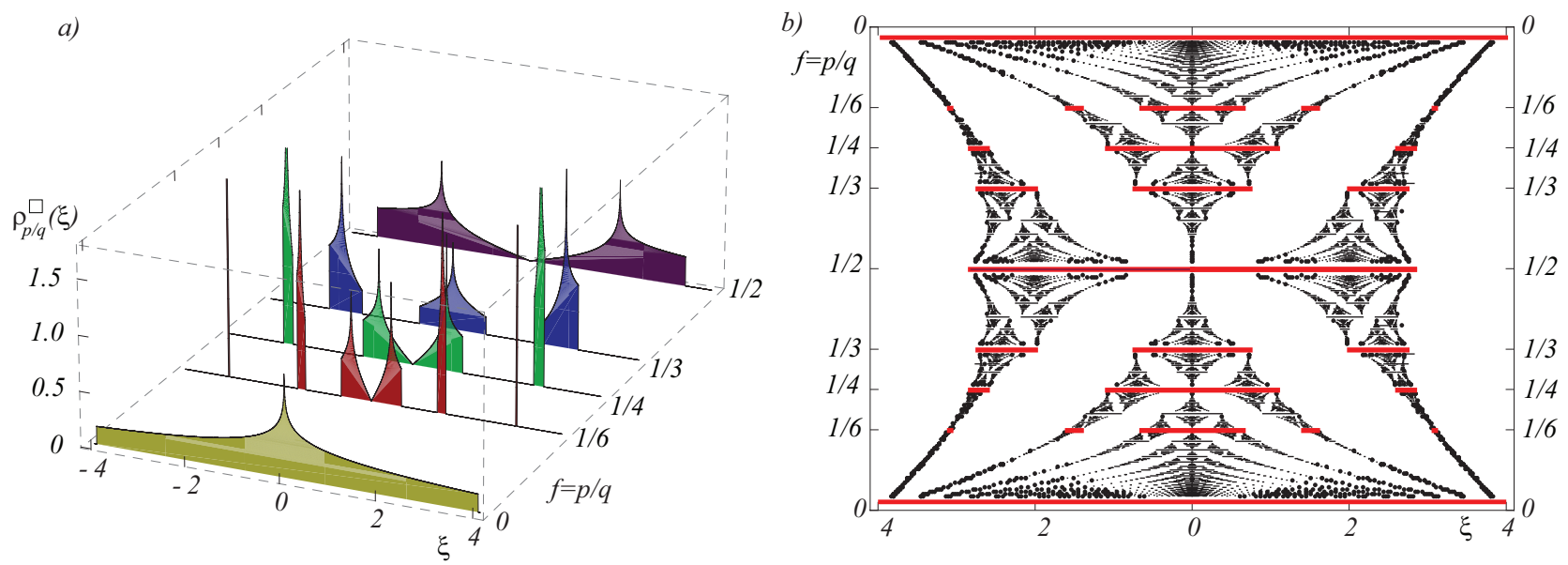

Figure 9: (Color online) a) The density of states for several values of the frustration parameters $f=0,1 / 2,1 / 3,1 / 4$, and $1 / 6$ in square lattice. b) The projection of the DOS on $\xi-f$ surface results in Hofstadter butterfly. Red lines correspond to our analytical results.

$$
\begin{gathered}
\times\left\{\sqrt{2} \sec \left(\varphi-\frac{\pi}{2}\right) \sqrt{\cos \left[\frac{1}{2}\left(\varphi+\frac{\pi}{4}\right)\right] \cos \left[\frac{1}{2}\left(\varphi-\frac{\pi}{4}\right)\right]}\left[\Theta\left(5+\sqrt{21}-\xi^{2}\right)-\Theta\left(6+2 \sqrt{3}-\xi^{2}\right)\right]\right. \\
+\sec \left(\varphi+\frac{\pi}{6}\right) \sqrt[4]{1-\sqrt{2} \cos \left(\varphi-\frac{\pi}{3}\right)-\frac{1}{2} \cos \left(2 \varphi+\frac{\pi}{3}\right)} \Theta\left(5-\sqrt{21}-\xi^{2}\right) \\
\left.+\sec \left(\varphi-\frac{\pi}{6}\right) \sqrt[4]{1-\sqrt{2} \cos \left(\varphi+\frac{\pi}{3}\right)-\frac{1}{2} \cos \left(2 \varphi-\frac{\pi}{3}\right)}\left[\Theta\left(6-2 \sqrt{3}-\xi^{2}\right)-\Theta\left(2-\xi^{2}\right)\right]\right\}, \\
\varphi=\frac{1}{3} \arctan \left(\frac{\left|\left(\xi^{4}-8 \xi^{2}+8\right) \sqrt{16+8 \xi^{2}-\xi^{4}}\right|}{\xi^{6}-12 \xi^{4}+24 \xi^{2}+32}\right) .
\end{gathered}
$$

The projection of the analytically calculated density of states for several values of rotation on the $\xi-f$ surface results in the Hofstadter butterfly (see Fig. 9). The calculations of the Hofstadter spectrum feasible for general purposes are impractical in applications since the phase boundary calculated from Eq. 31) strongly depends on the structure of DOS.

\section{Appendix C: Triangular lattice in rotating frame}

For the lattice topologically equivalent to triangular (Fig. 8) the Harper's equation takes form:

$$
\operatorname{det}\left(\begin{array}{ccccc}
M_{1} & N_{1} & \cdots & 0 & \bar{N}_{n} \\
\bar{N}_{1} & M_{2} & N_{2} & 0 & 0 \\
\vdots & \bar{N}_{2} & M_{3} & \ddots & 0 \\
0 & 0 & \ddots & \ddots & N_{n-1} \\
N_{n} & 0 & 0 & \bar{N}_{n-1} & M_{n}
\end{array}\right)=0
$$

where $n=q / 2$ for even and $n=q$ for odd $q$. Moreover

$$
\begin{aligned}
M_{n} & =-t_{\boldsymbol{k}}^{\triangle}-2 \cos \left(k_{2}+4 \pi f n\right), \\
N_{n} & =-e^{-i k_{1}}-e^{2 \pi f(2 n+1)} e^{i\left(k_{1}+k_{2}\right)},
\end{aligned}
$$

and $\bar{N}_{n}$ is a complex conjugation. Now the generalized structure factor can be written in form

$$
\gamma_{ \pm n}^{\triangle}=-\cos n k_{1}-\cos n k_{2} \mp \cos \left(n k_{1}+n k_{2}\right) .
$$

From the above we can derive the equations for the energy dispersion (see Table VI) for several values of the frustration rotation parameter. Analytical results sometimes come at a price of the complexity of solutions and that is the case here. Therefore we omit exact results for the dispersions $t_{\boldsymbol{k}}^{\triangle}$ and present them only in the simple case of $f=1 / 4$.

\section{Triangular lattice without rotation - $f=0$}

Cases with $f=0(1 / 3)$ and $f=1 / 2(1 / 6)$ are relatively simple since the density of states can be easily obtained 


\begin{tabular}{c|c|c}
\hline \hline$p$ & $q$ & $t_{\boldsymbol{k}} \equiv t_{\boldsymbol{k}}^{\triangle}$ \\
\hline 1 & 1 & $t_{\boldsymbol{k}}+2 \gamma_{1}^{\triangle}=0$ \\
\hline 1 & 2 & $t_{\boldsymbol{k}}-2 \gamma_{-2}^{\triangle}=0$ \\
\hline 1 & 3 & $t_{\boldsymbol{k}}^{3}-9 t_{\boldsymbol{k}}-6+2 \gamma_{3}^{\triangle}=0$ \\
\hline 1 & 4 & $t_{\boldsymbol{k}}^{2}-6-2 \gamma_{4}^{\triangle}=0$ \\
\hline 1 & 6 & $t_{\boldsymbol{k}}^{3}-9 t_{\boldsymbol{k}}+6-2 \gamma_{-6}^{\triangle}=0$ \\
\hline
\end{tabular}

Table VI: Energy dispersion for rotating triangular lattice for values $f=p / q$ used in calculations.

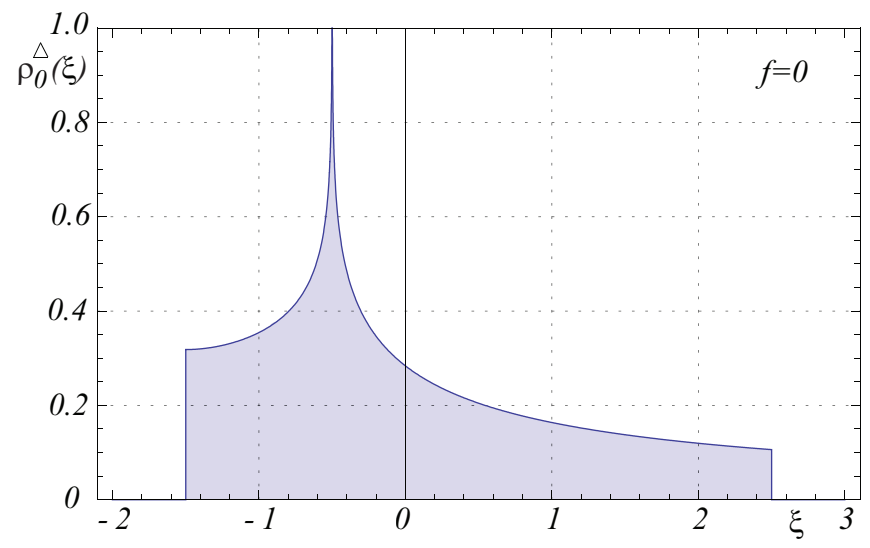

Figure 10: (Color online) DOS for triangular lattice without rotation $f=0$. by changing $\beta \rightarrow-\beta$ or equivalently $\xi \rightarrow-\xi$.

$$
\rho_{0}^{\triangle}(\xi) \equiv \rho(1,0.5, \xi)
$$

2. Rotating triangular lattice with $f=1 / 2$

$$
\rho_{1 / 2}^{\triangle}(\xi) \equiv \rho(1,-0.5, \xi)=\rho_{0}^{\triangle}(-\xi)
$$

3. Rotating triangular lattice with $f=1 / 3$

$$
\begin{aligned}
\rho_{1 / 3}^{\triangle}(\xi) & =2^{1 / 3} \sqrt{\xi^{2}-12}\left|\left(\xi^{2}-3\right) \Phi^{2}(\xi)\right| \rho_{0}^{\triangle}\left[\frac{1}{2}\left(6+9 \xi-\xi^{3}\right)\right] \\
& \times\left\{|\Phi(\xi)-6|^{-1}\left\{\Theta\left[\xi-2 \sqrt{3} \cos \left(\frac{\arctan \sqrt{107}}{3}\right)\right]-\Theta\left[\xi-2 \sqrt{3} \cos \left(\frac{\pi}{18}\right)\right]\right\}\right. \\
& +|-3(1-i \sqrt{3})-\Phi(\xi)|^{-1}\left\{\Theta\left[\xi+\theta\left(\frac{\arctan \sqrt{107}}{3}\right)\right]+\Theta\left[\xi+\theta\left(\frac{\pi}{18}\right)\right]\right\} \\
& \left.+|6-\Phi(\xi)|^{-1}\left\{\Theta\left[\xi+\theta\left(\frac{\arctan \sqrt{107}}{3}\right)\right]-\Theta\left[\xi+\theta\left(\frac{\pi}{18}\right)\right]\right\}\right\}
\end{aligned}
$$

where

$$
\Phi(\xi)=2^{1 / 3}\left[\xi\left(\xi^{2}-9\right)+\left(\xi^{2}-3\right) \sqrt{\xi^{2}-12}\right]^{2 / 3}
$$

and

$$
\theta(\varphi)=2 \sqrt{3} \sin \varphi+\sqrt{2 \sin ^{2} \varphi-2 \sqrt{3} \cos \varphi \sin \varphi+1}
$$

4. Rotating triangular lattice with $f=1 / 4$

The energy dispersion calculated from Harper's equation (B1) can be written in the form:

$$
t_{1 / 4}^{\triangle}=\left\{\begin{array}{lll}
+\sqrt{2\left(3+\gamma_{2}^{\triangle}\right)} & \text { for } & t_{\boldsymbol{k}}^{\triangle} \in[-\sqrt{11}, 0), \\
-\sqrt{2\left(3+\gamma_{2}^{\triangle}\right)} & \text { for } & t_{\boldsymbol{k}}^{\triangle} \in[0, \sqrt{11}] .
\end{array}\right.
$$




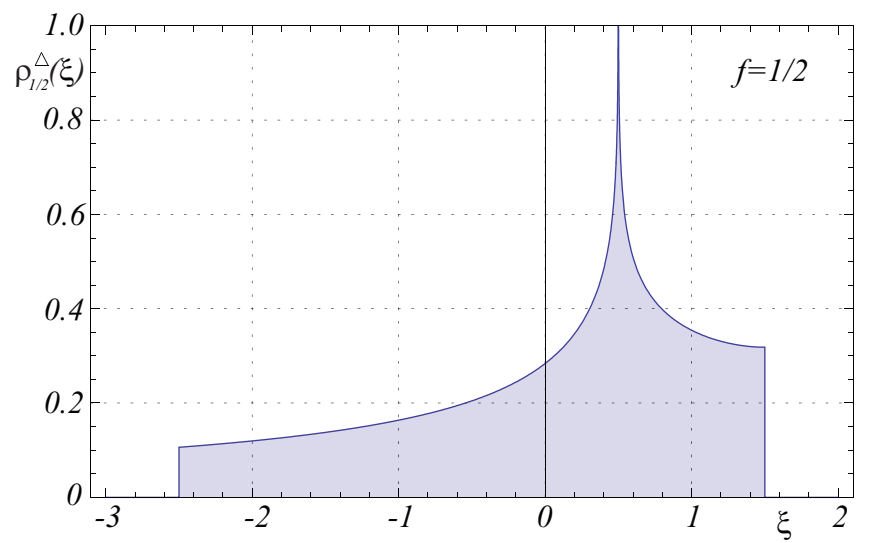

Figure 11: (Color online) DOS for rotating triangular lattice $f=1 / 2$.

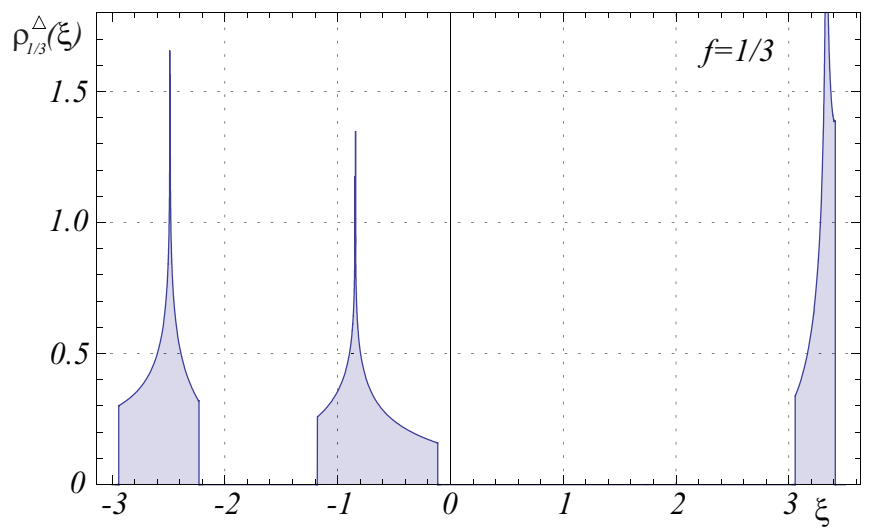

Figure 12: (Color online) DOS for rotating triangular lattice $f=1 / 3$.
Integrating over the wave vectors belonging to the Brillouin zone, we obtain

$$
\rho_{1 / 4}^{\triangle}(\xi)=\frac{|\xi|}{2} \rho_{0}^{\triangle}\left[-\frac{1}{2}\left(\xi^{2}-6\right)\right] .
$$

$$
\rho_{1 / 6}^{\triangle}(\xi)=\rho_{1 / 3}^{\triangle}(-\xi)
$$

1 M. H. Anderson, J. R. Ensher, M. R. Matthews, C. E. Wieman and E. A. Cornell, Science 269, 198 (1995).

2 D. Jaksch, C. Bruder, J. I. Cirac, C. W. Gardiner, and P. Zoller, Phys. Rev. Lett. 81, 3108 (1998).

3 M. Greiner, O. Mandel, T. Esslinger, T. W. Hänsch, and I. Bloch, Nature 415, 39 (2002).

4 M. Greiner, I. Bloch, O. Mandel, T. W. Hänsch and T. Esslinger, Phys. Rev. Lett. 87, 160405 (2001).

${ }^{5}$ I. Coddington, P. C. Haljan, P. Engels, V. Schweikhard, S. Tung, and E. A. Cornell, Phys. Rev. A 70, 063607 (2004).

6 S. Tung, V. Schweikhard, and E. A. Cornell, Phys. Rev. Lett. 97, 240402 (2006).

7 V. Schweikhard, S. Tung, and E. A. Cornell, Phys. Rev. Lett. 99, 030401 (2007).

${ }^{8}$ R. Bhat, B. M. Peden, B. T. Seaman, M. Krämer, L. D. Carr, and M. J. Holland, Phys. Rev. A 74, 063606 (2006).

9 R. Bhat, M. Krämer, J. Cooper, and M. J. Holland, Phys. Rev. A 76, 043601 (2007).

10 N. R. Cooper, Advances in Physics 57, 539 (2008).

11 A. Leggett, Quantum liquids (Oxford, New York, 2006).

12 F. Chevy and J. Dalibard, Europhysics News 37, 12 (2006).
13 M. R. Matthews, B. P. Anderson, P. C. Haljan, D. S. Hall, C. E. Wieman, and E. A. Cornell, Phys. Rev. Lett. 83, 2498 (1999).

14 J. R. Abo-Shaer, C. Raman, J. M.Vogels and W. Ketterle, Science 292, 476 (2001).

15 E. Hodby, G. Hechenblaikner, S. A. Hopkins, O. M. Maragó, and C. J. Foot, Phys. Rev. Lett. 88, 010405 (2002).

16 P. C. Haljan, I. Coddington, P. Engels, and E. A. Cornell, Phys. Rev. Lett. 87, 210403 (2001).

17 V. Bretin, S. Stock, Y. Seurin, and J. Dalibard, Phys. Rev. Lett. 92, 050403 (2004).

18 R. O. Umucalilar, M. Öktel, Phys. Rev. B 76, 055601 (2007).

19 D. S. Goldbaum and E. J. Mueller, Phys. Rev. A 77, 033629 (2008).

20 T. K. Kopeć, Phys. Rev. B 70, 054518 (2004).

21 T. P. Polak and T. K. Kopeć, Phys. Rev. B 76, 094503 (2007).

22 M. P. A. Fisher, P. B. Weichman, G. Grinstein, and D. S. Fisher, Phys. Rev. B 40, 546 (1989). 


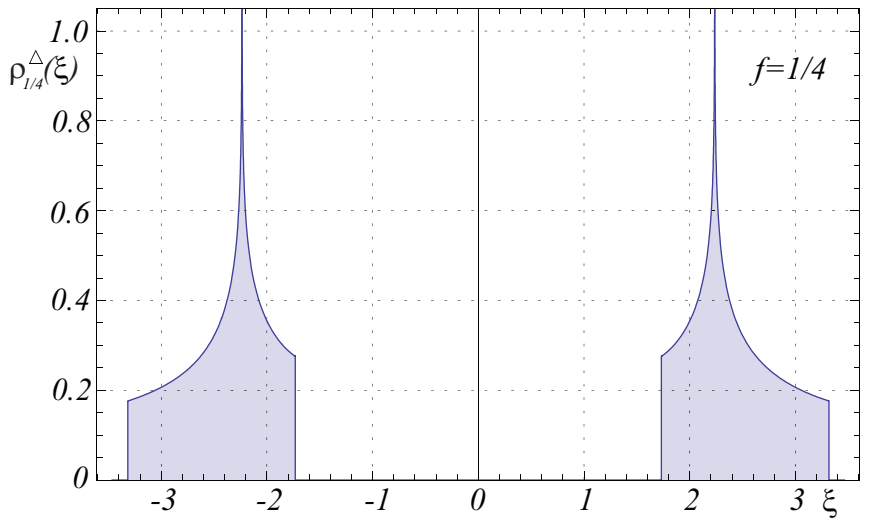

Figure 13: (Color online) DOS for rotating triangular lattice $f=1 / 4$.

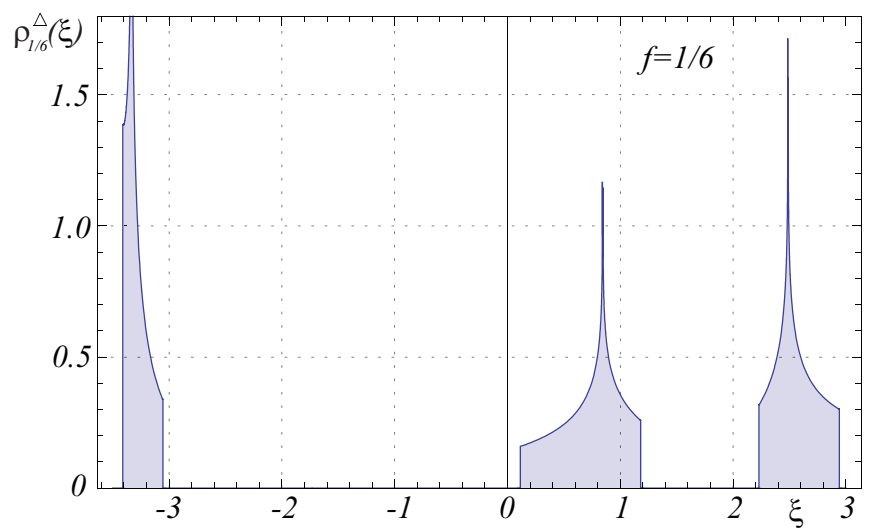

Figure 14: (Color online) DOS for rotating triangular lattice $f=1 / 6$.
23 A. P. Kampf, G. T. Zimanyi, Phys. Rev. B 47, 279 (1993).

24 T. K. Kopeć, J. V. José, Phys. Rev. B 60, 7473 (1999).

25 B. Capogrosso-Sansone, Ş. Güneş Söyler, Nikolay Prokof'ev and B. Svistunov, Phys. Rev. A 77, 015602 (2008).

26 N. Teichmann, D. Hinrichs, M. Holthaus, and A. Eckardt, Phys. Rev. B 79, 100503 (2009).

27 M. Ö. Oktel, M. Niţă, and B. Tanatar, Phys. Rev. B 75, 045133 (2007).

28 M. Niemeyer, J. K. Freericks, and H. Monien, Phys. Rev. B 60, 2357 (1999).

29 N. D. Mermin and H. Wagner, Phys. Rev. Lett. 17, 1133 (1966).

30 D. R. Hofstadter, Phys. Rev. B 14, 2239 (1976).

31 Y. Hasegawa, P. Lederer, T. M. Rice, and P. B. Wiegmann, Phys. Rev. Lett. 63, 907 (1989).

32 M. Abramovitz and I. Stegun, Handbook of Mathematical Functions (Dover, New York, 1970). 